\title{
Transient Lesion of the Splenium of the Corpus Callosum after Acute Ischemic Stroke
}

\author{
Yoshitaka Yamaguchi ${ }^{1,2}$, Yuko Iwasaki ${ }^{2}$, Manabu Wada ${ }^{1}$, Naoki Makita ${ }^{2}$, Hikaru Nagasawa ${ }^{1}$, \\ Tatsushi Yamakawa ${ }^{1}$ and Kazunori Toyoda ${ }^{2}$
}

\begin{abstract}
:
Two patients who showed transient lesions in the splenium of the corpus callosum (SCC) secondary to acute ischemic stroke are reported. Both patients had embolic strokes and showed an isolated lesion in the SCC on magnetic resonance imaging (MRI) 1-2 weeks after the onset of stroke, with a hyperintense lesion on diffusion-weighted imaging and decreased apparent diffusion coefficient values, with no symptoms related to the lesion. In both cases, the lesion disappeared on MRI approximately 1 week later. Clinicians should note that transient SCC lesions can occur following acute ischemic stroke and avoid misdiagnosing them and performing unnecessary examinations or treatment.
\end{abstract}

Key words: acute ischemic stroke, magnetic resonance imaging, mild encephalitis/encephalopathy with a reversible splenial lesion, splenium of the corpus callosum, transient lesion in the splenium of the corpus callosum

(Intern Med 58: 1011-1015, 2019)

(DOI: 10.2169/internalmedicine.1010-18)

\section{Introduction}

A transient lesion in the splenium of the corpus callosum (SCC) observed on magnetic resonance imaging (MRI) is rarely encountered in clinical practice (1). Although the precise etiology of the lesion has not been well clarified, it has been reported in a wide range of clinical conditions. On the other hand, no cases of transient lesions in SCC related to acute ischemic stroke have been reported to date. Thus, two cases that showed transient lesions in the SCC secondary to acute ischemic stroke are herein reported.

\section{Case Reports}

\section{Case 1}

A 41-year-old man was admitted to our hospital because of consciousness disturbance and sensory aphasia. Diffusionweighted imaging (DWI) on day 4 showed hyperintensity in the left temporal and parietal lobes (Fig. 1A, B). DWI, fluid-attenuated inversion-recovery (FLAIR), and the apparent diffusion coefficient (ADC) showed no SCC abnormalities (Fig. 1B-D). Digital subtraction angiography showed occlusion of the left middle cerebral artery at the M2 proximal segment (Fig. 1E). There was no atherosclerotic change and there were no findings suggestive of intracranial artery dissection. The patient was diagnosed as having an embolic stroke and was treated with intravenous argatroban, edaravone, and oral aspirin. Carotid ultrasound, transthoracic echocardiography, and Holter electrocardiography showed no evidence of an embolic source. The patient would not consent to undergo any further examinations, including transesophageal echocardiography and long-term Holter electrocardiography. A diagnosis of embolic stroke of undetermined source was made; however, the precise etiology of his ischemic stroke remained unclear. Although his symptoms were unchanged, MRI on day 13 showed an isolated lesion in the SCC that appeared hyperintense lesion on DWI and FLAIR. The lesion showed no enhancement on contrastenhanced imaging (not shown), and decreased ADC values (Fig. 2A-C). Laboratory tests, including a hemogram, renal

${ }^{1}$ Department of Neurology, Yamagata Prefectural Central Hospital, Japan and ${ }^{2}$ Department of Cerebrovascular Medicine, National Cerebral and Cardiovascular Center, Japan

Received: February 13, 2018; Accepted: September 18, 2018; Advance Publication by J-STAGE: November 19, 2018

Correspondence to Dr. Yoshitaka Yamaguchi, y.yamaguchi830@gmail.com 

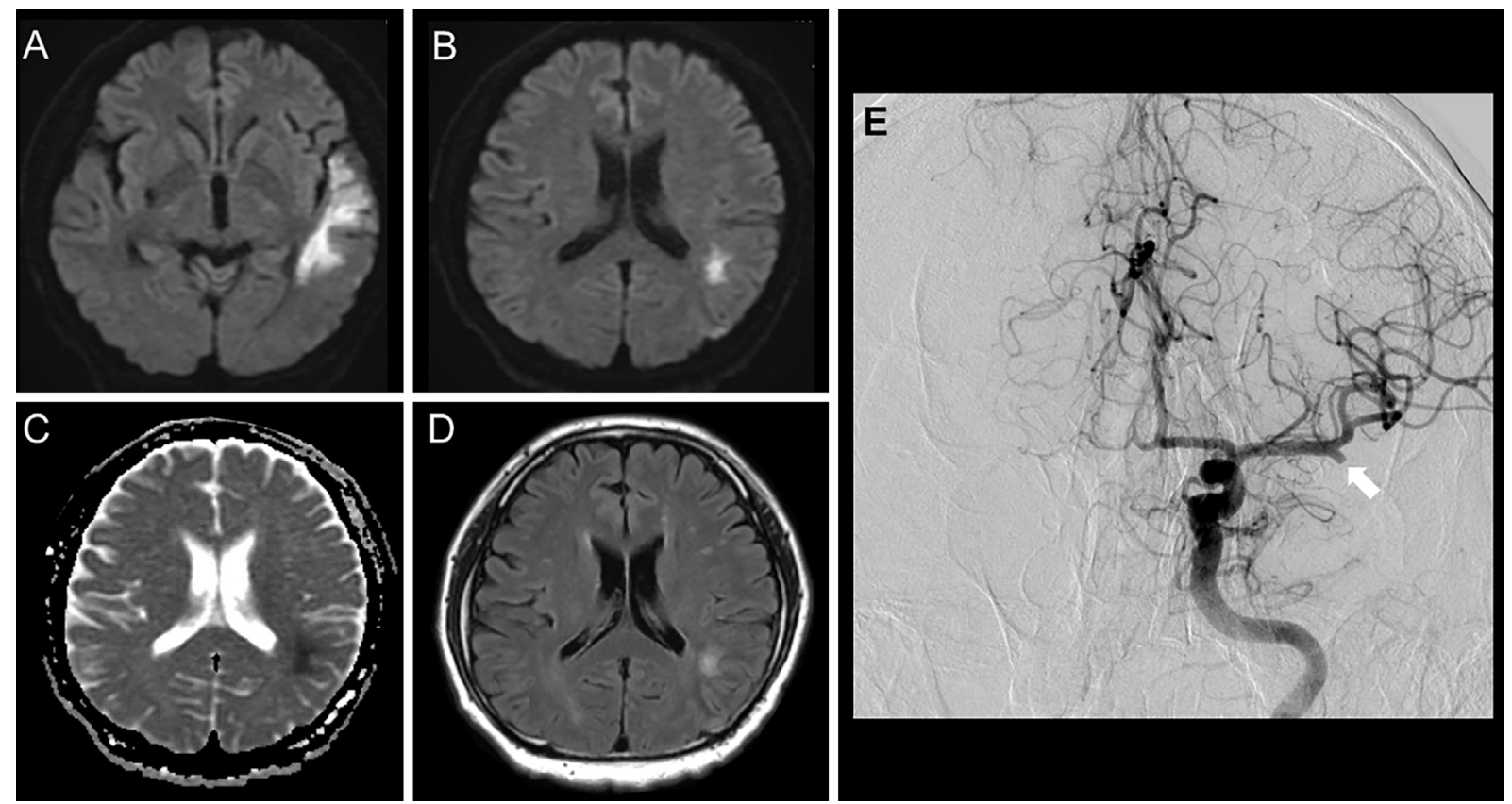

Figure 1. Magnetic resonance imaging and digital subtraction angiography of Case 1. DWI (A, B), ADC mapping (C), and FLAIR (D) on Day 4. Right internal carotid arteriography (E) showed occlusion of the left middle cerebral artery at the M2 proximal segment (arrow). No lesion was present in the SCC (B-D). ADC: apparent diffusion coefficient, DWI: diffusion-weighted imaging, FLAIR: fluid-attenuated inversion-recovery, SCC: splenium of the corpus callosum
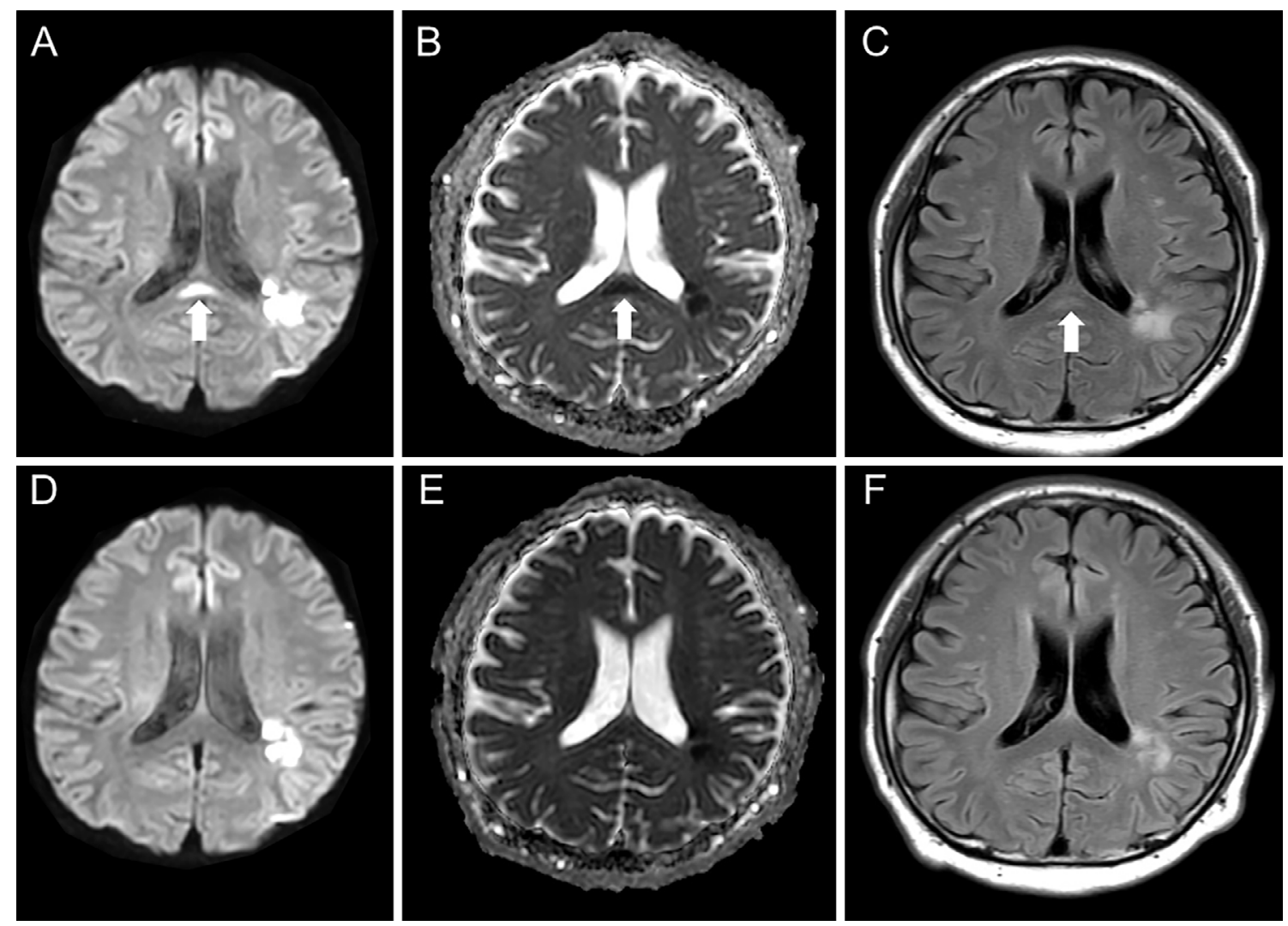

Figure 2. Follow-up magnetic resonance imaging of Case 1. An SCC lesion with hyperintensity on DWI (A), FLAIR (C), and decreased ADC values (B) were seen on Day 13 (arrow). The lesion disappeared on DWI (D), ADC mapping (E), and FLAIR (F) on Day 20. ADC: apparent diffusion coefficient, DWI: diffusion-weighted imaging, FLAIR: fluid-attenuated inversion-recovery, SCC: splenium of the corpus callosum 

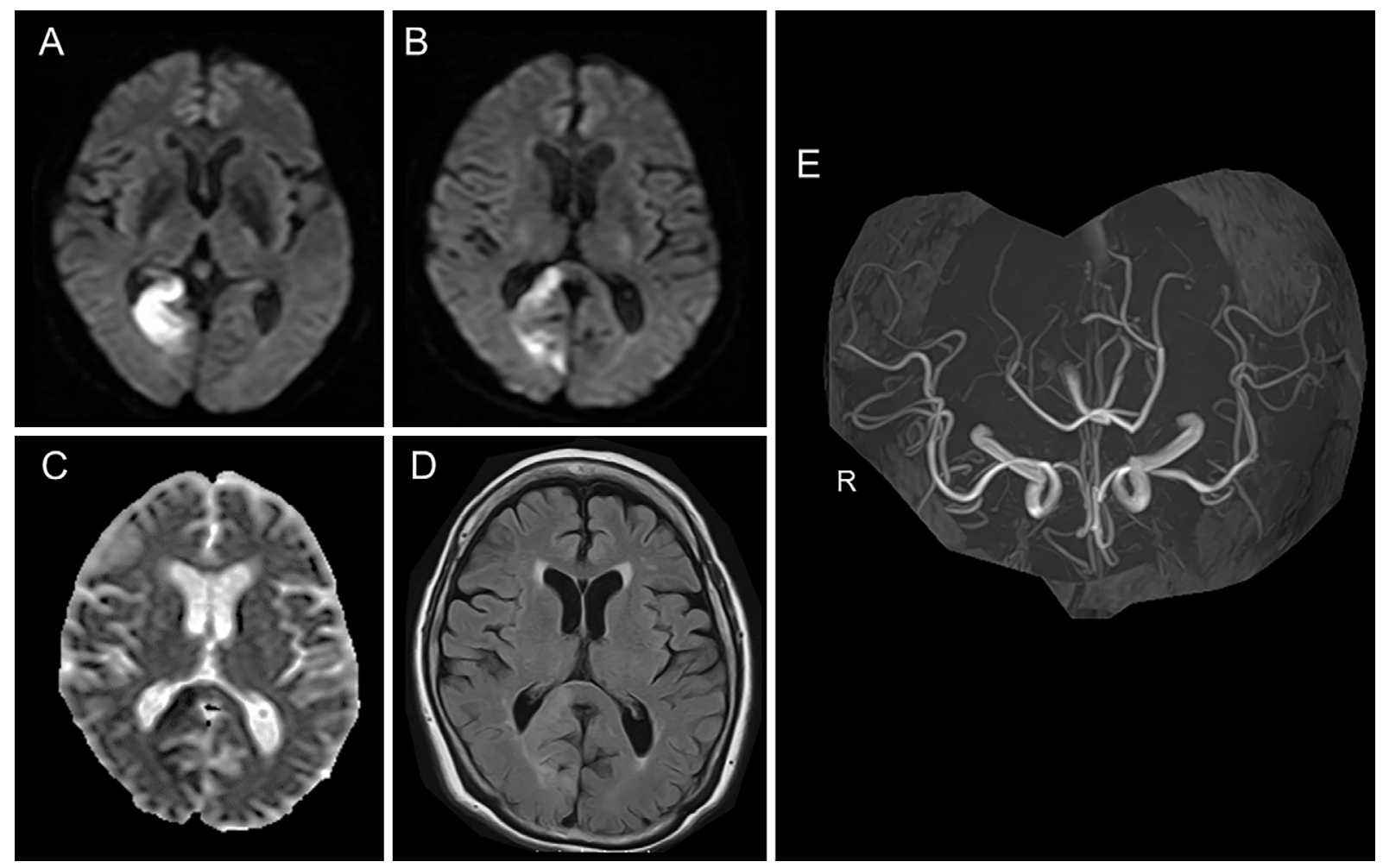

Figure 3. Magnetic resonance imaging of Case 2. DWI (A, B), ADC mapping (C), FLAIR (D), and MR angiography (E) on Day 2. No lesion was present on the left side of the SCC (B-D). ADC: apparent diffusion coefficient, DWI: diffusion-weighted imaging, FLAIR: fluid-attenuated inversion-recovery, MRA: magnetic resonance angiography, SCC: splenium of the corpus callosum

and liver function tests, and serum electrolyte and cerebrospinal fluid analyses revealed values that were within normal limits. MRI on day 20 showed that the SCC abnormalities had disappeared (Fig. 2D-F); thus, the lesion was diagnosed as a transient lesion of the SCC.

\section{Case 2}

An 81-year-old woman was admitted to our hospital because of left hemianopia. DWI and FLAIR on Day 2 showed areas of hyperintensity on the right side of the SCC and the right occipital lobe (Fig. 3A, B). There were no DWI, ADC, and FLAIR abnormalities on the left side of the SCC (Fig. 3B-D). MR angiography showed no occlusive or stenotic lesions (Fig. 3E). From her medical history of paroxysmal atrial fibrillation, she was diagnosed as having a cardiogenic embolism. She was treated with intravenous heparin, which was converted to oral apixaban. Although her symptoms were unchanged, MRI on day 7 showed a hyperintense lesion on DWI and FLAIR, with decreased ADC values that extended to the left side of the SCC (Fig. 4A-C). The values of laboratory tests, including a hemogram, renal and liver function tests, and a serum electrolyte analysis were within normal limits. MRI on day 15 showed significantly decreased signal intensity on DWI and FLAIR on the left side of the SCC, whereas the high intensity on DWI and FLAIR (due to infarction) remained on the right side of the SCC (Fig. 4D-F). Thus, the lesion that extended from the right to the left side of the SCC was considered to be a transient lesion of the SCC.

\section{Discussion}

A transient lesion of the SCC has been reported in various clinical conditions, including infectious encephalitis/encephalopathy [alternatively known as mild encephalitis/encephalopathy with a reversible splenial lesion (MERS)] (1), epilepsy or antiepileptic drug withdrawal (2), metabolic disturbances such as hypoglycemia (3), and status migrainosus (4). To the best of our knowledge, this is the first report to demonstrate transient SCC lesions after acute ischemic stroke.

Because data - especially from pathological investigations -have been lacking due to the benign nature of transient SCC lesions and the complete reversal of the neurological findings, neither the exact pathophysiology nor the specific site predilection of transient SCC lesions has been clarified. The most likely explanations for these transient lesions of the SCC have been thought to be rapidly resolving intramyelinic edema or the influx of inflammatory cells and macromolecules, combined with related cytotoxic edema (1). Because the SCC lesions in the present cases were asymptomatic, and because no clinical manifestations related to the transient SCC lesions were present, the possible explanations for the occurrence of the transient SCC lesions are controversial.

Wallerian degeneration (WD), a degenerative phenomenon 

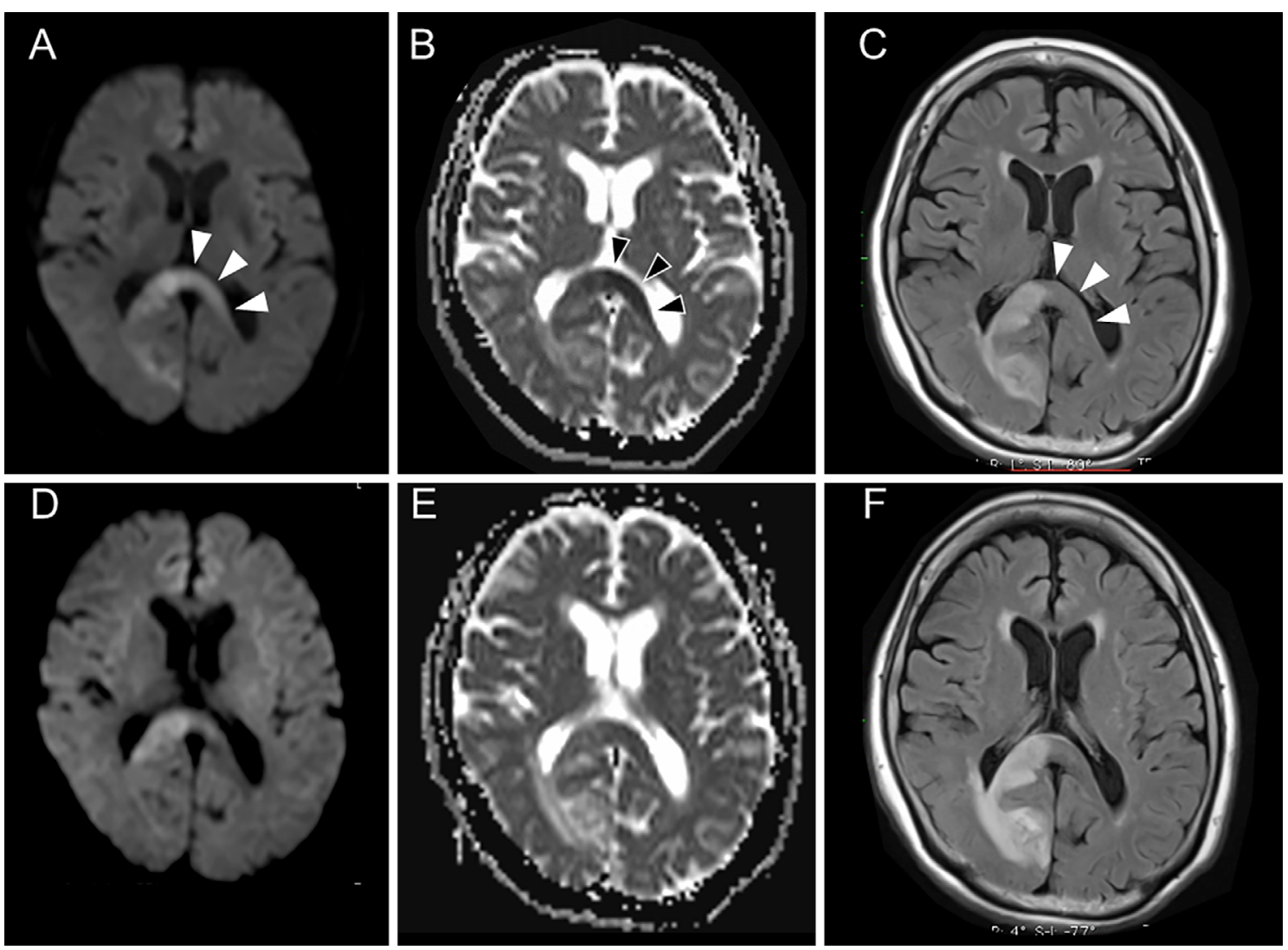

Figure 4. Follow-up magnetic resonance imaging of Case 2. A hyperintense lesion on DWI (A) and FLAIR (C) with decreased ADC values (B) extended to the left side of the SCC on Day 7 (arrowheads). This lesion on the left side of the SCC became obscure on DWI (D), ADC mapping (E), and FLAIR (F) on day 15. ADC: apparent diffusion coefficient, DWI: diffusion-weighted imaging, FLAIR: fluid-attenuated inversion-recovery, SCC: splenium of the corpus callosum

that affects the nerve fibers distal to a primary lesion, is characterized by a stereotypical course, starting with the disintegration of the axonal structures within days after injury, followed by the degradation of myelin and atrophy of the affected fiber tracts (5). WD after ischemic stroke is a wellrecognized phenomenon not only in the corticospinal tracts (6), but also in the SCC (7-9). Because the radiological findings of WD are recognized in the chronic stage and are usually irreversible, the clinical and radiological features of the SCC lesions in the present cases were different from those of WD.

Reversible cerebral vasoconstriction syndrome (RCVS) is a clinical condition characterized by thunderclap headache and non-aneurysmal segmental cerebral vasoconstriction on arteriography. RCVS is often accompanied by vasogenic edema in the cerebral white matter, which can be considered posterior reversible encephalopathy syndrome (PRES) (10). RCVS or PRES can be an important etiology of SCC lesions (8). In contrast to the SCC lesions of the present two cases, the ADC values usually increase in PRES lesions, suggesting the presence of vasogenic edema due to dysfunction of the cerebrovascular autoregulation mechanism. Furthermore, clinical features related to PRES, including headache, seizure, consciousness disturbance, encephalopathy, visual disturbance, and potential causes of PRES (including severe hypertension and exposure to immunosuppressants) were lacking in the two present cases. Moreover, there were no angiographic findings suggesting vasospasm in the two present cases. To the best of our knowledge, there have been no reports describing RCVS or PRES with a single lesion localized in the SCC.

The corpus callosum is a bundle of axons of neurons projecting from one side of the brain to the other and consists almost entirely of white matter. Oligodendrocytes enwrap neuronal axons with myelin, providing electrical insulation of axons and facilitation of nerve impulse stimulation. Electrical stimulation, mediated by oligodendrocyte direct axonprecursor cell contacts and diffusible substances, including neurotransmitters, promotes the proliferation and differentiation of oligodendrocyte precursor cells (OPCs), resulting in the synthesis of myelin in the corpus callosum (11). The examination of mouse brain slices has demonstrated that the pattern of neuronal activity determines the functional changes triggered at the synapses between axons and OPCs; temporary increases of quantal glutamate release have been observed after the cessation of stimulation (11). Because the SCC has connections to the temporal, parietal, and occipital lobes $(12,13)$, acute infarctions in these lobes may cause the isolation of neuronal activity from the affected lobes to the SCC. This might result in a disturbance of synaptic con- 
nections with a temporary increase of glutamate release in the synapses between axons and OPCs in the SCC, and subsequent glutamatergic toxicity in glial cells (14) and the secondary intensity change on DWI seen in the present cases. Similar transient signal changes on DWI have been reported in the substantia nigra of patients with striatal infarction (15). The mechanism of the signal change was considered to be a transsynaptic in nature: the loss of an inhibitory $\gamma$-aminobutyric acidergic output from the striatum to the substantia nigra results in excessive excitation, causing neuronal damage in the substantia nigra (15). Awareness of transient SCC lesions is important because they must be differentiated from other diseases, including infectious and non-infectious conditions. It might be misdiagnosed as encephalitis/encephalopathy or a recurrence of ischemic stroke and, as a result, mislead clinicians who may then perform unnecessary examinations or treatment.

\section{Conclusion}

Two cases involving patients with transient SCC lesions secondary to acute ischemic stroke were presented. Clinicians should note that transient SCC lesions can occur following acute ischemic stroke.

The authors state that they have no Conflict of Interest (COI).

\section{References}

1. Tada H, Takanashi J, Barkovich AJ, et al. Clinically mild encephalitis/encephalopathy with a reversible splenial lesion. Neurology 63: 1854-1858, 2004.

2. Gürtler S, Ebner A, Tuxhorn I, Ollech I, Pohlmann-Eden B, Woermann FG. Transient lesion in the splenium of the corpus callosum and antiepileptic drug withdrawal. Neurology 65: 10321036, 2005.

3. Ma JH, Kim YJ, Yoo WJ, et al. MR imaging of hypoglycemic encephalopathy: lesion distribution and prognosis prediction by diffusion-weighted imaging. Neuroradiology 51: 641-649, 2009.
4. Samanta D. Transient lesion in the splenium of the corpus callosum in status migrainosus. Acta Neurol Belg 115: 397-398, 2015.

5. Johson AC, Mcnabb AR, Rossiter RJ. Chemistry of wallerian degeneration; a review of recent studies. Arch Neurol Psychiatry 64: 105-121, 1950.

6. Sawlani V, Gupta RK, Singh MK, Kohli A. MRI demonstration of Wallerian degeneration in various intracranial lesions and its clinical implications. J Neurol Sci 146: 103-108, 1997.

7. Gupta RK, Saksena S, Hasan KM, et al. Focal Wallerian degeneration of the corpus callosum in large middle cerebral artery stroke: serial diffusion tensor imaging. J Magn Reson Imaging 24: 549555, 2006.

8. Park SE, Choi DS, Shin HS, et al. Splenial lesions of the corpus callosum: disease spectrum and MRI findings. Korean J Radiol 18: 710-721, 2017.

9. Gandhi K, Gillihan L, Wozniak MA, Zhuo J, Raghavan P. Progressive Wallerian degeneration of the corpus callosal splenium in a patient with alexia without agraphia: advanced MR findings. Neuroradiol J 27: 653-656, 2014.

10. Ducros A. Reversible cerebral vasoconstriction syndrome. Lancet Neurol 11: 906-917, 2012.

11. Nagy B, Hovhannisyan A, Barzan R, Chen TJ, Kukley M. Different patterns of neuronal activity trigger distinct responses of oligodendrocyte precursor cells in the corpus callosum. PLoS Biol 15: e2001993, 2017.

12. Park HJ, Kim JJ, Lee SK, et al. Corpus callosal connection mapping using cortical gray matter parcellation and DT-MRI. Hum Brain Mapp 29: 503-516, 2008.

13. Hofer S, Frahm J. Topography of the human corpus callosum revisited--comprehensive fiber tractography using diffusion tensor magnetic resonance imaging. Neuroimage 32: 989-994, 2006.

14. Simantov R. Glutamate neurotoxicity in culture depends on the presence of glutamine: implications for the role of glial cells in normal and pathological brain development. J Neurochem 52: 1694-1699, 1989.

15. Nakajima M, Hirano T, Terasaki T, Uchino M. Signal change of the substantia nigra on diffusion-weighted imaging following striatal infarction. Intern Med 49: 65-68, 2010.

The Internal Medicine is an Open Access journal distributed under the Creative Commons Attribution-NonCommercial-NoDerivatives 4.0 International License. To view the details of this license, please visit (https://creativecommons.org/licenses/ by-nc-nd/4.0/).

(C) 2019 The Japanese Society of Internal Medicine Intern Med 58: 1011-1015, 2019 